\title{
The Diagnostic Challenge of Seronegative Autoimmune Encephalitis With Super-Refractory Status Epilepticus
}

\author{
Koti Vadagandla $^{1}$, Vinay Jahagirdar ${ }^{2}$, Kaanthi Rama ${ }^{2}$ \\ 1. Anesthesia, Medicover Hospital, Hyderabad, IND 2. Medicine, Gandhi Medical College and Hospital, Secunderabad, \\ IND
}

Corresponding author: Vinay Jahagirdar, vinayjaha@gmail.com

\begin{abstract}
Autoimmune encephalitis is an immune-mediated syndrome, with sub-acute to chronic presentations, such as memory impairment, altered sensorium, behavioral abnormality, psychosis, and seizures. It poses a twofold diagnostic challenge: firstly, because of its variable clinical presentation and secondly, due to the wide variety of autoimmune antibodies causing it, which makes it difficult to identify the underlying etiology. Treatment should not be delayed due to pending laboratory workup, as early recognition and initiation of therapy prevents long term neurological sequelae. This is a case report of a 59-year-old female who presented with neuropsychiatric symptoms, which evolved into refractory status epilepticus and autonomic dysfunction, requiring anesthesia induced coma. While her MRI had positive findings of encephalitis, she tested negative for infectious diseases and antibody panels.
\end{abstract}

Review began 10/24/2020 Review ended 11/06/2020 Published 11/20/2020

\section{() Copyright 2020}

Vadagandla et al. This is an open access article distributed under the terms of the Creative Commons Attribution License CC-BY 4.0., which permits unrestricted use, distribution, and reproduction in any medium, provided the original author and source are credited.
Categories: Anesthesiology, Internal Medicine, Neurology

Keywords: autoimmune, encephalitis, status epilepticus, seronegative encephalitis, epileptic seizures, refractory seizure

\section{Introduction}

Autoimmune encephalitis syndromes have a wide clinical spectrum ranging from typical limbic encephalitis to syndromes with complex neuropsychiatric symptoms, such as the loss of memory, cognition, psychosis, seizures, abnormal movements, or coma. Its etiology can be broadly divided into three groups. In the first group, antibodies are produced against tumor antigens in a paraneoplastic syndrome. In the second group, antibodies are produced against extracellular and intracellular ion channels and proteins. In the third group, antigens are not clearly established.

Imaging abnormalities include medial temporal lobe changes and contrast-enhancing abnormalities in cortical or subcortical regions. Electroencephalography (EEG) shows infrequent epileptic activity but a frequent, slow, disorganized activity that does not correlate with most abnormal movements. A unique EEG pattern called extreme delta brush is seen in prolonged illness [1].

Seronegative autoimmune encephalitis is a subcategory of autoimmune encephalitis diagnosed when autoimmune antibodies are not detected in cerebrospinal fluid (CSF) or serum [2-3]. The possible reasons for the absence of antibodies, as stated by Najjar et al., include declining serum antibodies and the existence of unidentified antibodies which are yet to be discovered [2]. Early recognition and treatment prevent relapse and reduce long-term neurological sequelae, as proposed by Darnell and Posner [4]. Management of autoimmune encephalitis is mainly by using immunosuppressants. First-line therapy includes steroids and intravenous immunoglobulins (IVIG). Second-line immunotherapy, such as rituximab or cyclophosphamide, should be considered if the symptoms do not subside with the first-line therapy.

\section{Case Presentation}

A 59-year-old female patient with no significant past medical history presented to the emergency room with a history of fever, headache, drowsiness, and body pains for one week. Suspecting viral encephalitis, acyclovir was initiated. The patient had one episode of generalized tonic-clonic seizures (GTCS) postadmission. Magnetic resonance imaging (MRI) of the brain ruled out a cerebrovascular accident. CSF analysis did not show features of meningoencephalitis. Serum sodium was $122 \mathrm{meq} / \mathrm{mol}$. Hyponatremia was suspected as the cause of the GTCS, and the patient was started on 3\% normal saline. The patient recovered, and a Glasgow Coma Scale score of 15 was noted. However, six hours later, she became drowsy and went into respiratory failure with a partial pressure of carbon dioxide $\left(\mathrm{PaCO}_{2}\right)$ of 55, prompting non-invasive ventilatory support. The patient had one more episode of GTCS. On examination following that episode, she had altered sensorium, disorientation, confusion, and faciobrachial dystonic seizures (FBDS), hinting at autoimmune encephalitis. A serum autoimmune panel was ordered, which came back negative. Three antiepileptic drugs, namely, sodium valproate, phenytoin, and levetiracetam, were started. However, the patient continued to have recurrent complex partial seizures. She developed hyperthermia, tachycardia, and 


\section{Cureus}

hypertension, indicating autonomic dysfunction. Infectious causes, including herpes simplex, chikungunya, cytomegalovirus, and dengue were ruled out. Procalcitonin was normal. The autonomic dysfunction was treated with nitroglycerine and dexmedetomidine infusion. Despite this, the patient had continuous faciobrachial dystonia and cataplexy. Methylprednisolone, $1 \mathrm{~g} /$ day, was started. Paraneoplastic causes were ruled out through CT scans and tumor markers. The patient required ventilator support because of recurrent status epilepticus and autonomic instability. As she continued to have recurrent seizures despite steroids, intravenous immunoglobulins were initiated. The patient had persistent seizures on the ventilator with four antiepileptic drugs (levetiracetam, phenytoin, sodium valproate, clonazepam), and propofol infusion of 1.5 $\mathrm{mg} / \mathrm{kg} / \mathrm{hr}$. A diagnosis of super-refractory status epilepticus was made because of persistent seizures after 24 hrs of propofol infusion. Repeat MRI of the brain showed medial temporal lobe hyperintensity. Thiopentone, $3 \mathrm{mg} / \mathrm{kg} / \mathrm{hr}$, was started for burst suppression and the dosage was increased to a maximum of $6 \mathrm{mg} / \mathrm{kg} / \mathrm{hr}$. Midazolam, $2 \mathrm{mg} / \mathrm{hr}$, was continued, along with thiopentone, and an intermittent bolus of thiopentone was given.

After three days of thiopentone infusion, the EEG showed a generalized, reduced spike pattern. The thiopentone dose was reduced and eventually stopped, but she continued to have faciobrachial dystonia. Suspecting N-methyl-D-aspartate (NMDA)-receptor encephalitis, magnesium sulfate, $1 \mathrm{~g} / \mathrm{hr}$, and ketamine infusion, $1.5 \mathrm{mg} / \mathrm{kg} / \mathrm{hr}$, were started and were continued for one week. The FBDS persisted, prompting the initiation of second-line immunotherapy with rituximab, $625 \mathrm{mg}$ (at $375 \mathrm{mg} / \mathrm{m}^{2}$ ) with serial CD19 and CD20 antibody monitoring.

The patient improved neurologically with reduced spikes on EEG. Eventually, two weeks down the line, the patient was discharged without any neurological or cognitive abnormality.

The investigations and timeline of events during the hospital stay are depicted in Tables 1-2, respectively.

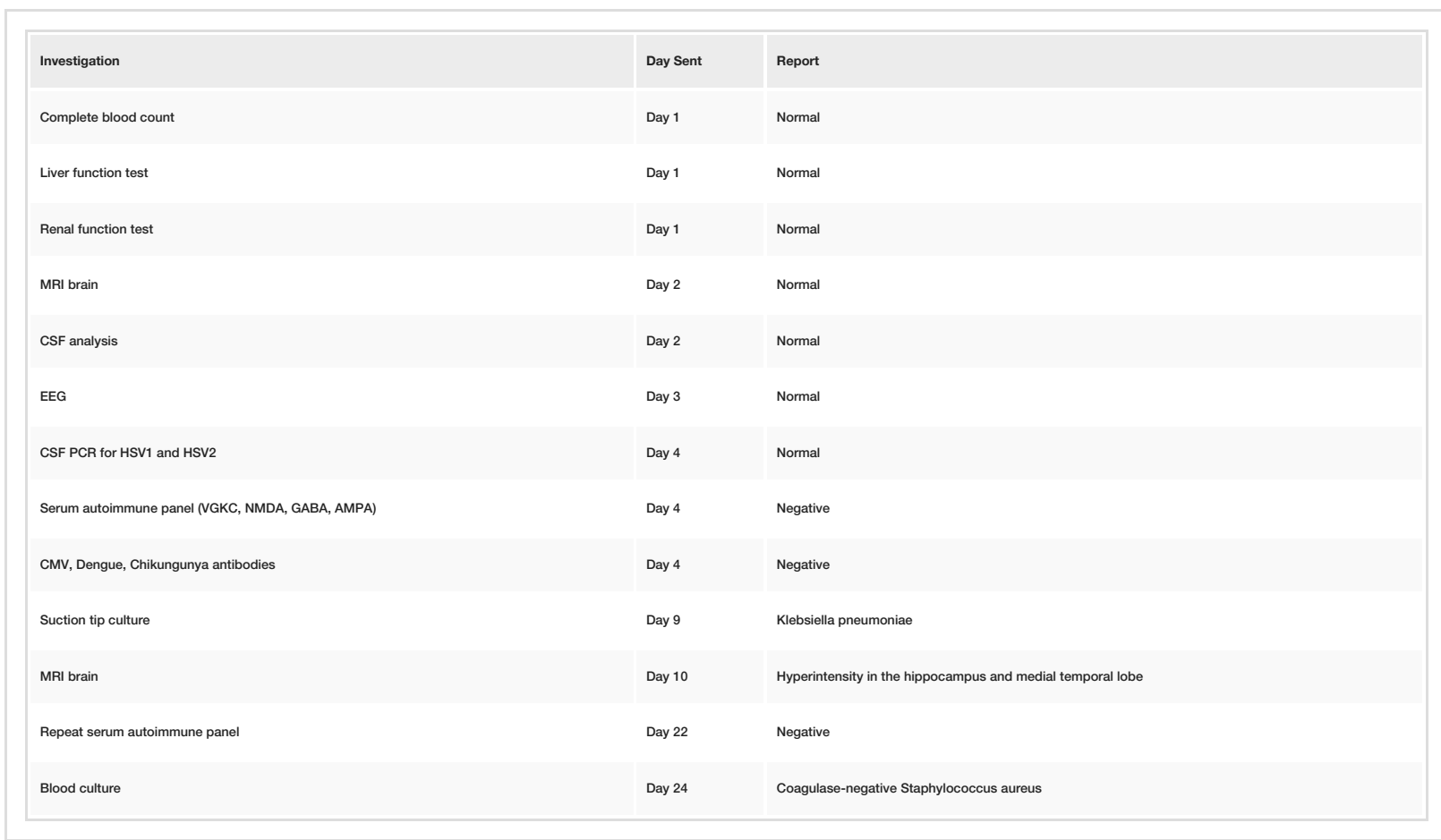

\section{TABLE 1: Chronology of Laboratory and Imaging Investigations Ordered}

AMPA: a-amino-3-hydroxy-5-methyl-4-isoxazolepropionic acid: CMV: cytomegalovirus; CSF: cerebrospinal fluid; EEG: electroencephalography; GABA: gamma-aminobutyric acid; HSV1: herpes simplex virus 1; HSV2: herpes simplex virus 2; MRI: magnetic resonance imaging; NMDA: N-methylD-aspartate; PCR: polymerase chain reaction; VGKC: voltage-gated potassium channel 


\section{Cureus}

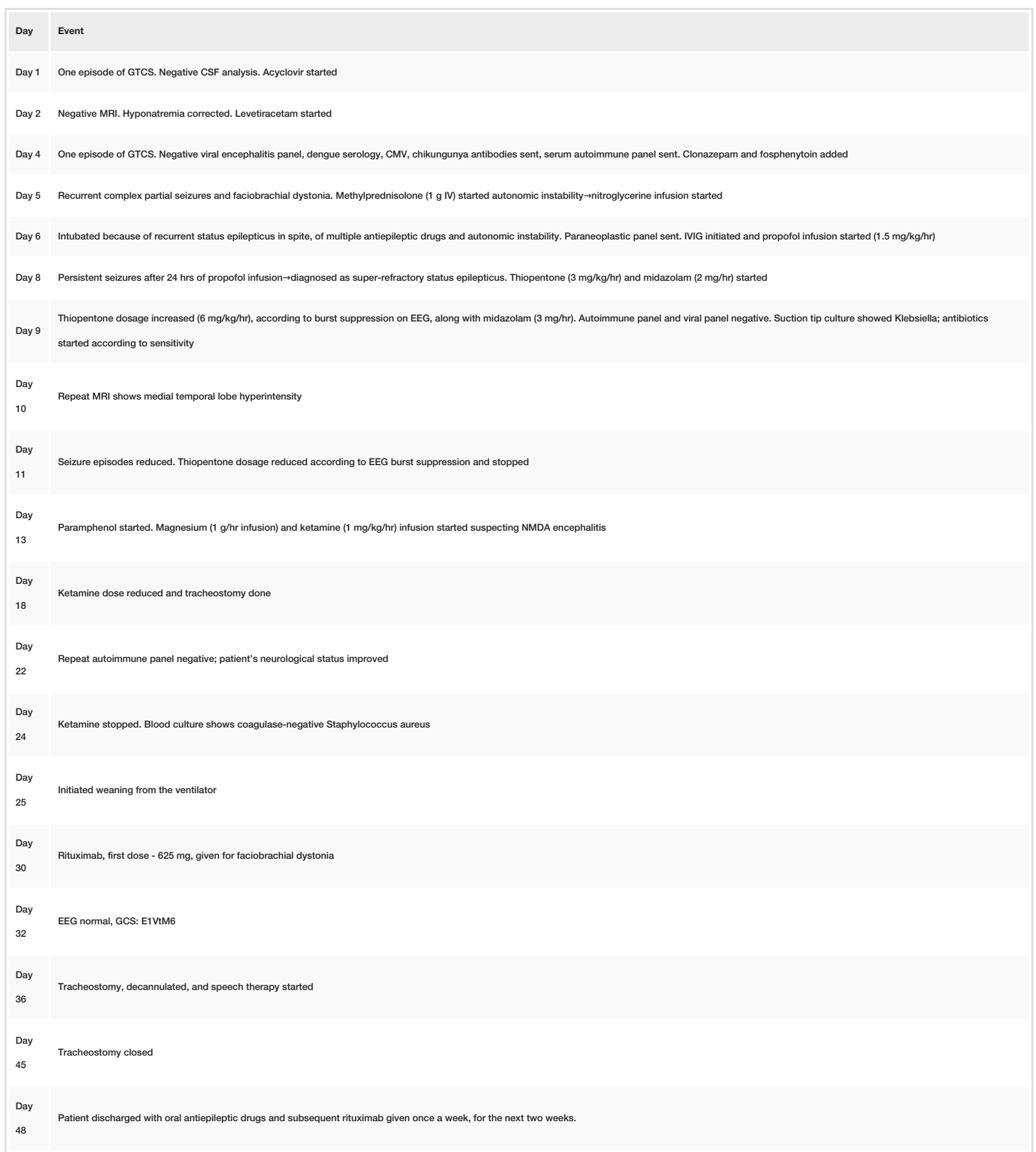

\section{TABLE 2: Summary of Clinical Events}

CMV: cytomegalovirus; CSF: cerebrospinal fluid; EEG: electroencephalography; GCS: Glasgow Coma Scale; GTCS: generalized tonic-clonic seizure; IV: intravenous; IVIG: intravenous immunoglobulin G; MRI: magnetic resonance imaging; NMDA: N-methyl-D-aspartate;

\section{Discussion}

The term seronegative autoimmune encephalitis has been used to describe a subgroup of autoimmune encephalitis cases with negative serologic tests for autoantibodies [2-3].

Pathophysiology of the disease can be explained by the presence of a diverse group of autoantibodies to intracellular or extracellular receptors and paraneoplastic tumor antigens, such as anti-Hu antibodies and anti-NMDA receptor antibodies [5]. There are many such unidentified receptors as reported by previous case reports [2-3]. Paraneoplastic syndromes should be considered as etiology if the patient is diagnosed to have certain tumors, evidenced by imaging and tumor markers. Some tumors associated with paraneoplastic encephalitis include immature teratoma in females and thymoma [5]. The tumor antigens act as receptors for antibodies in such cases [6-7]. 
Clinical presentation in autoimmune encephalitis varies based on the causative antibody [5]. Psychiatric manifestations and abnormal movements are the early presenting features in most patients. There are instances where a patient was referred to a psychiatrist in view of predominant psychiatric manifestations, leading to a delay in diagnosis, as well as early initiation of treatment which may result in persistent disabilities.

Patients with positive antibodies to gamma-aminobutyric acid (GABA) and NMDA present with seizures and refractory status epilepticus, as mentioned by Lancaster et al. and Petit-Pedrol et al. [8-9]. FBDS are classically associated with leucine-rich glioma-inactivated 1 (LGI1) antibody [10]. Our case presented with psychiatric manifestations and behavioral abnormalities. She tested negative for GABA antibodies but developed super-refractory status epilepticus and FBDS later. Status epilepticus was earlier defined as lasting for 30 minutes or longer but is now more often defined as lasting five minutes or longer [11]. Refractory status epilepticus is defined as persistent seizures, despite the use of two intravenous medications, one of which is a benzodiazepine [12]. Super-refractory status epilepticus is defined as status epilepticus that continues or recurs 24 hours or more after the onset of anesthetic therapy. Despite aggressive treatment with four anti-epileptics and an anesthesia-induced coma, the seizures lasted for more than 48 hours in our case.

Existing criteria for autoimmune encephalitis depend on antibody testing, which may lead to a delay in diagnosis. To overcome this, Graus et al. have suggested the criteria presented in Table 3, for autoantibodynegative but probable autoimmune encephalitis [13].

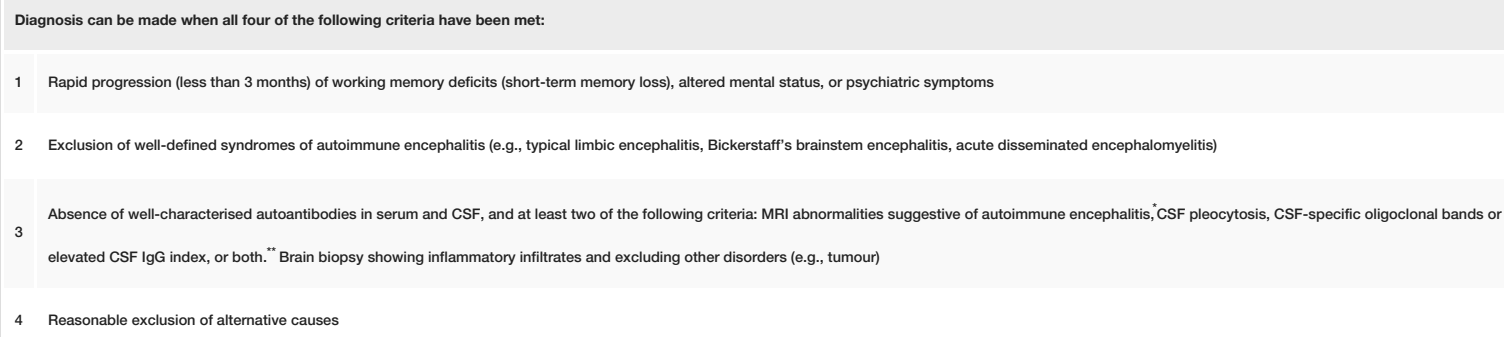

\section{TABLE 3: Criteria for Autoantibody-negative but Probably Autoimmune Encephalitis}

* autoimmune encephalitis can occur with normal or atypical MRI findings

*^ absence of pleocytosis does not rule out autoimmune encephalitis

CSF: cerebrospinal fluid; IgG: immunoglobulin G

Graus et al. [13]

Our patient underwent a serum antibody panel twice to check for antibodies to NMDA, GABA, and voltagegated potassium channels (VGKC). However, the panel was negative both times. Other possible factors, including infectious, metabolic causes (like hyponatremia), and paraneoplastic tumor syndromes were ruled out. MRI of the brain showed medial temporal hyperintensity in T2 films, as documented in other similar case reports [8, 14-15]. This eventually led us to a diagnosis of seronegative autoimmune encephalitis. Constant EEG monitoring helped to monitor our case and direct treatment. EEG monitoring is necessary to exclude subclinical seizures, for prognosis, and also for management based on burst suppression [16].

Early diagnosis and intervention can improve prognosis and prevent relapses in autoimmune encephalitis [4]. So far, there is no convincing evidence to show whether IVIG or plasmapheresis is superior to the other for management of autoimmune encephalitis, and hence either may be used. If the patient remains significantly impaired after first-line therapy, second-line treatments are deployed. Second-line therapies include rituximab (often $375 \mathrm{mg} / \mathrm{m}^{2}$ weekly for four weeks) or cyclophosphamide (750 mg/m² IV monthly until improvement is noted). Rituximab has a good safety profile compared to cyclophosphamide. There are no existing indications or guidelines regarding the timing of second-line immunotherapy. Our patient received Solu-Medrol ${ }^{\circledR}$ (methylprednisolone), $1 \mathrm{~g} /$ day for five days, and IVIG, $0.4 \mathrm{~g} / \mathrm{kg}$ for five days. Due to persistent faciobrachial dystonia, rituximab was started as second-line therapy after 19 days of completion of the IVIG course.

\section{Conclusions}

Autoimmune encephalitis is a spectrum of disease with neurological and psychiatric symptoms. The 
presentation may vary based on the type of antibodies causing it. In our case, the main concerning issue was super-refractory status epilepticus, requiring continuous anesthesia to suppress EEG burst activity. This poses a diagnostic challenge as present laboratory techniques lack specificity to identify this. There are many unidentified intracellular and extracellular protein receptors; hence, a negative serology panel for antibodies should not delay the initiation of primary immunomodulatory therapy (steroids, plasmapheresis). A strong clinical suspicion of disease, after a panel to rule out paraneoplastic and infectious serology, supported by MRI imaging, is the mainstay to identify seronegative autoimmune encephalitis. Primary therapy should be initiated to prevent disease progression. If the patient continues to have symptoms despite steroids and IVIG, then second-line immunotherapy with agents like rituximab can be used. Our patient recovered well after second-line immunotherapy and was discharged without any cognitive or neurological symptoms.

\section{Additional Information \\ Disclosures}

Human subjects: Consent was obtained by all participants in this study. Conflicts of interest: In compliance with the ICMJE uniform disclosure form, all authors declare the following: Payment/services info: All authors have declared that no financial support was received from any organization for the submitted work. Financial relationships: All authors have declared that they have no financial relationships at present or within the previous three years with any organizations that might have an interest in the submitted work. Other relationships: All authors have declared that there are no other relationships or activities that could appear to have influenced the submitted work.

\section{Acknowledgements}

We thank Dr. Indira Gauri Tati for her unwavering help and support.

\section{References}

1. Schmitt SE, Pargeon K, Frechette ES, Hirsch LJ, Dalmau J, Friedman D: Extreme delta brush: a unique EEG pattern in adults with anti-NMDA receptor encephalitis. Neurology. 2012, 79:1094-1100. 10.1212/WNL.0b013e3182698cd8

2. Najjar S, Pearlman D, Zagzag D, Devinsky O: Spontaneously resolving seronegative autoimmune limbic encephalitis. Cogn Behav Neurol. 2011, 24:99-105. 10.1097/WNN.0b013e3182248193

3. Storey K, Matěj R, Rusina R: Unusual association of seronegative, nonparaneoplastic limbic encephalitis and relapsing polychondritis in a patient with history of thymectomy for myasthenia: a case study. J Neurol. 2011, 258:159-161. 10.1007/s00415-010-5691-4

4. Darnell RB, Posner JB: A new cause of limbic encephalopathy. Brain. 2005, 128:1745-1746. 10.1093/brain/awh592

5. Lancaster E: The diagnosis and treatment of autoimmune encephalitis . J Clin Neurol. 2016, 12:1-13. 10.3988/jcn.2016.12.1.1

6. Dalmau J, Furneaux HM, Rosenblum MK, Graus F, Posner JB: Detection of the anti-Hu antibody in specific regions of the nervous system and tumor from patients with paraneoplastic encephalomyelitis/sensory neuronopathy. Neurology. 1991, 41:1757-1764. 10.1212/WNL.41.11.1757

7. Dalmau J, Tüzün E, Wu HY, et al.: Paraneoplastic anti-N-methyl-D-aspartate receptor encephalitis associated with ovarian teratoma. Ann Neurol. 2007, 61:25-36. 10.1002/ana.21050

8. Lancaster E, Lai M, Peng X, et al.: Antibodies to the GABA(B) receptor in limbic encephalitis with seizures: case series and characterisation of the antigen. Lancet Neurol. 2010, 9:67-76. 10.1016/S14744422(09)70324-2

9. Petit-Pedrol M, Armangue T, Peng X, et al.: Encephalitis with refractory seizures, status epilepticus, and antibodies to the GABAA receptor: a case series, characterisation of the antigen, and analysis of the effects of antibodies. Lancet Neurol. 2014, 13:276-286. 10.1016/S1474-4422(13)70299-0

10. Irani SR, Michell AW, Lang B, et al.: Faciobrachial dystonic seizures precede Lgi1 antibody limbic encephalitis. Ann Neurol. 2011, 69:892-900. 10.1002/ana.22307

11. Nelson SE, Varelas PN: Status epilepticus, refractory status epilepticus, and super-refractory status epilepticus. Continuum (Minneap Minn). 2018, 24:1683-1707. 10.1212/CON.0000000000000668

12. Marawar R, Basha M, Mahulikar A, Desai A, Suchdev K, Shah A: Updates in refractory status epilepticus . Crit Care Res Pract. 2018, 2018:9768949. 10.1155/2018/9768949

13. Graus F, Titulaer MJ, Balu R, et al.: A clinical approach to diagnosis of autoimmune encephalitis . Lancet Neurol. 2016, 15:391-404. 10.1016/S1474-4422(15)00401-9

14. Dalmau J, Lancaster E, Martinez-Hernandez E, Rosenfeld MR, Balice-Gordon R: Clinical experience and laboratory investigations in patients with anti-NMDAR encephalitis. Lancet Neurol. 2011, 10:63-74. 10.1016/S1474-4422(10)70253-2

15. Lancaster E, Huijbers MG, Bar V, et al.: Investigations of caspr2, an autoantigen of encephalitis and neuromyotonia. Ann Neurol. 2011, 69:303-311. 10.1002/ana.22297

16. Sutter R, Kaplan PW, Cervenka MC, Thakur KT, Asemota AO, Venkatesan A, Geocadin RG: Electroencephalography for diagnosis and prognosis of acute encephalitis . Clin Neurophysiol. 2015, 126:1524-31. 10.1016/j.clinph.2014.11.006 